\title{
Wide-band X-ray Studies of Inner Disc Structure in Galactic Black Hole Binaries
}

\section{Megumi Shidatsu}

Department of Astronomy, Kyoto University

E-mail: Shidatsudkusastro.kyoto-u.ac.jp

\section{Yoshihiro Ueda}

Department of Astronomy, Kyoto University, Japan

Takafumi Hori

Department of Astronomy, Kyoto University, Japan

\section{Chris Done}

Department of Physics, Durham University, UK

\section{Satoshi Nakahira}

ISS Science Project Office, Institute of Space and Astronautical Science (ISAS),

Japan Aerospace Exploration Agency (JAXA), Japan

\begin{abstract}
We present our recent results from Suzaku and Swift Time-Of-Opportunity observations of the Galactic black hole transients MAXI J1305-704, H 1743-322, and 4U 1630-472. The wideband high-quality data obtained in the low/hard state (the former two sources) and in the very high state (4U 1630-472) enable us to constrain the structure of the accretion flow in various Xray luminosities. We find that all the spectra, including the 4U 1630-472 data, taken 4 days after the detection of the baryonic jet []] , contain strong Comptonised components and suggest disc truncation. In the H 1743-322 data, a low-frequency QPO is detected, whose frequency is found to correlate the X-ray luminosity and photon index. This may be explained by the evolution of the disc truncation radius. Considering these results, we discuss the inner disc structure of black hole binaries over a wide range of mass accretion rates.
\end{abstract}

Swift: 10 Years of Discovery,

2-5 December 2014

La Sapienza University, Rome, Italy 


\section{Introduction}

Transient black hole X-ray binaries (BHXBs) in our galaxy provide important clues to understand the fundamental physics of black hole accretion. They exhibit several distinct "states" with different X-ray spectral shapes at different luminosities. The most canonical ones among them are so-called the "low/hard state", which is seen at relatively low luminosities during the beginning and end of outbursts, and the "high/soft state", appearing at higher soft X-ray ( $<10 \mathrm{keV})$ luminosities. At around the peak luminosities in outbursts, the "very high state" is sometimes observed, where the soft and hard X-ray fluxes are both strong.

The spectral shape is believed to reflect the geometry of the inner accretion discs, which differs significantly in each state (see [[13], [四], for recent reviews). In the high/soft state, the Xray spectrum is dominated by a soft X-ray component below $10 \mathrm{keV}$, which is well modelled by the thermal emission from the standard disc [एप]. BHXBs in the low/hard state show a hard, power-law shaped spectrum with a photon index of $<2$ and with an exponential cutoff at $\approx 100 \mathrm{keV}$, which generally described as the soft X-ray photons from the standard disc Compton-scattered by thermal electrons in hot inner flow or corona. The geometry and physical properties of the hot electron cloud are not well understood, however. The very high state is seen in high mass accretion rates near the Eddington limit, in which both the disc and Comptonisation components strongly contribute to the $\mathrm{X}$-ray spectra. The structure of the inner accretion flow in this state is poorly studied, because this state appears only in a short time in an outburst, and is difficult to observe.

In order to study the inner disc structure, we performed Suzaku observations of the Galactic BHXBs H 1743-322, MAXI J1305-704 in the low/hard state and 4U 1630-472 in the very high state. Here we report the results of the spectral and timing analysis of these Suzaku data and compare them with the high/soft state data obtained with Swift/XRT.

\section{Suzaku Observations of H 1743-322 in the Low/hard State}

H 1743-322 is a Galactic transient black hole binary. We carried out Suzaku target-ofopportunity (ToO) observations of the source in the low/hard state on 2012 October 4, 10, and 12 (hereafter Epoch-1, -2, and -3, respectively), when it became active.

Figure $\square$ plots the spectra in the individual epochs. The overall spectral shape is not largely changed in the three epochs, while it became slightly harder and fainter in Epoch-2 and -3 than in Epoch-1. The spectra are approximated by a power-law with a photon index of $\approx 1.6$, indicating that the source was in the low/hard state. We apply a physical model to the spectra, considering the general picture of the low/hard state. We use the diskbb model [10] as the direct emission from the standard disc and the nthcomp model as the Comptonisation. To account for the reflection component, the ireflect model [ए2] is convolved to nthcomp. A Gaussian component is combined to include the iron K- $\alpha$ emission line associated with the reflection continuum. The kdblur model [W] is also incorporated to model the relativistic blurring by the accretion disc around the black hole. Figure $\square$ shows the best-fit model for Epoch-1.

Assuming an isotropic Comptonised corona and the conservation of the total number of photons from the accretion disc, the inner disc radius can be derived from the intrinsic disc luminosity (see Equation (A1) in [8] ). The inner disc radii in the three epochs are estimated as $R_{\text {in }}=$ 


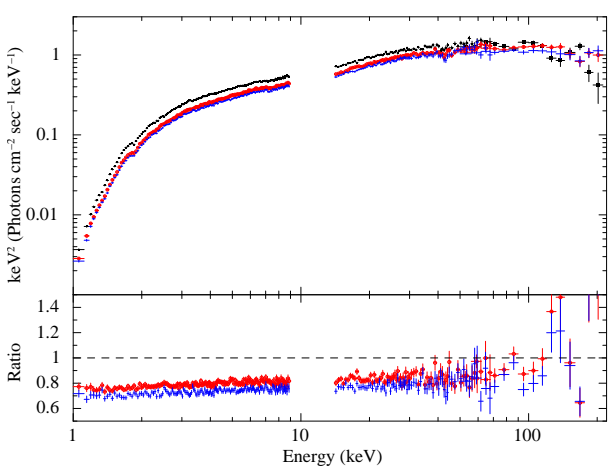

Figure 1: (Top) Time-averaged H 1743-322 spectra in Epoch-1 (black, filled square), Epoch-2 (red, open circle), and Epoch-3 (blue, cross). (Bottom) The spectral ratios of the second and third epochs with respect to the first one.

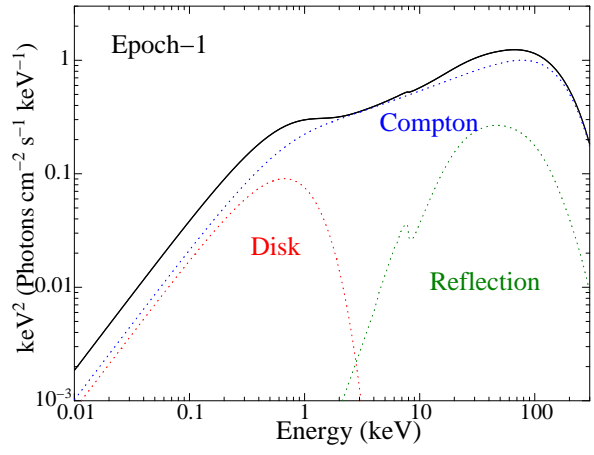

Figure 2: The best-fit Epoch-1 model in the $v F_{v}$ form, with contributions of each component separately plotted. Almost the same model is obtained as the best-fit models of the other two epochs. See [2]] for more detail.



Figure 3: Normalized PDSs in the individual epochs created from the Suzaku light curves in $14-50 \mathrm{keV}$ with $0.1 \mathrm{sec}$ bins. White noise is subtracted.

$120_{-11}^{+12} D_{8.5}\left(\cos i / \cos 75^{\circ}\right)^{-1 / 2} \mathrm{~km}, R_{\mathrm{in}}=141_{-17}^{+28} D_{8.5}\left(\cos i / \cos 75^{\circ}\right)^{-1 / 2} \mathrm{~km}$, and $R_{\text {in }}=110_{-12}^{+23}$ $D_{8.5}\left(\cos i / \cos 75^{\circ}\right)^{-1 / 2} \mathrm{~km}$ (where $D_{8.5}$ represents the distance in units of $8.5 \mathrm{kpc}$ ) for Epoch-1, -2, and -3 , respectively ${ }^{1}$. These values are 1.3-2.3 times larger than the inner radius in the high/soft state $\left(R_{\text {in }}=64 \pm 2 D_{8.5}\left(\cos i / \cos 75^{\circ}\right)^{-1 / 2} \mathrm{~km}[\right.$ [U] $)$. This suggests that the standard disc do not extend to the inner most stable circular orbit (ISCO) during the low/hard state observations.

Figure B 3 shows the normalized power density spectra (PDSs) in the three epochs. A weak, low frequency quasi periodic oscillation (QPO) is also detected in each epoch. Fitting it with a Gaussian model, we estimate the central frequency of the QPO as $0.206 \pm 0.003 \mathrm{~Hz}$ in Epoch-1, which become 1.4 times lower in Epoch-2 and Epoch-3 $(0.147 \pm 0.004 \mathrm{~Hz}$ and $0.141 \pm 0.004 \mathrm{~Hz}$, respectively) in accordance with the spectral hardening and the $\approx 20 \%$ decrease of the hard X-ray luminosity in the $15-50 \mathrm{keV}$ band.

The observed correlation of the QPO frequency with the X-ray luminosity and spectral hard-

\footnotetext{
${ }^{1}$ Here we considered the boundary condition and spectral hardening, and multiplied a representative correction factor, 1.19 [四].
} 
ness can be explained with the disc truncation and Lense-Thirring precession of hot inner flow extended between the black hole and the inner edge of the standard disc [6]. In the truncation disc model, the inner radius recedes as the mass accretion rate is decreased (see e.g., [䧃]). At the same time, the power of seed photons illuminating the hot flow becomes weaker, making the observed $\mathrm{X}$-ray spectrum harder. The decrease of the LF-QPO frequency can be explained if it reflects the timescale at the inner radius, where the standard disc is replaced to the hot flow. Although the inner disc radii that we estimated from the Suzaku data in each epoch have large uncertainties, they are compatible to the anti-correlation trend between the QPO frequency and the inner disc.

\section{Suzaku and Swift Observations of MAXI J1305-704: Comparison of the Low/hard and High/soft States}

MAXI J1305-704 is an X-ray transient discovered on 2012 with MAXI/GSC. [17] monitored its spectrum using MAXI and suggested that the source is likely a black hole X-ray binary. Swift/XRT detected strong ionized absorption lines, likely originated from the disc wind [1[4]. Chandra/HETG resolved complex absorption feature around $1 \mathrm{keV}$, which can be reproduced by ionized iron-L absorption lines [ए5]].

To investigate the detailed properties of the accretion flow, dips, and ionized absorbers in a low mass accretion rate, we observed MAXI J1305-704 during the low/hard state with Suzaku in 2012 July. We also analyzed the data obtained in the Swift/XRT observation from 2012 April 19 to 21 in the high/soft state [14] to compare with the Suzaku results. Here we focus on the inner disc structure. We refer the readers to [20] for details of the absorption dips and ionized absorption.

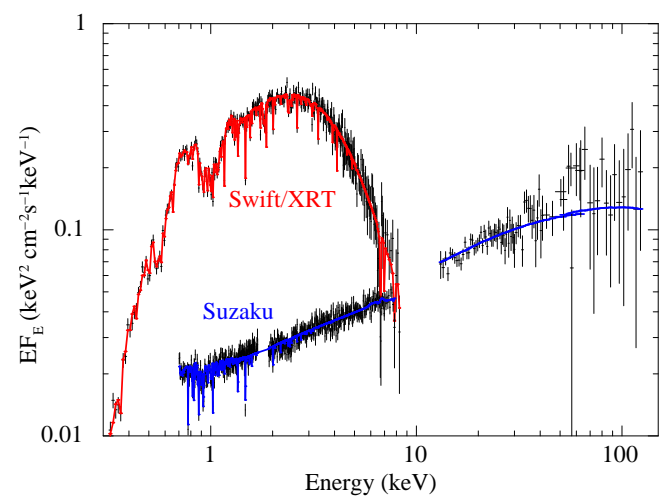

Figure 4: The time-averaged Suzaku spectrum of MAXI J1305-704 in the non-dip (persistent) periods during low/hard state, compared with the Swift/XRT non-dip spectrum in the high/soft state. The best-fit model of each spectrum is also plotted.

The time-averaged non-dip Suzaku spectrum in the low/hard state is characterized by a powerlaw component extended up to $130 \mathrm{keV}$ with a photon index of $\approx 1.6$, while the non-dip Swift/XRT spectrum in the high/soft state is well described by an multi-color disc component with an inner temperature of $\approx 1.0 \mathrm{keV}$. Both of them are affected by neutral and ionized absorptions. Figure $\mathbb{\theta}$ plots the Suzaku and Swift/XRT spectra and their best-fit model. Using the disk luminosity, we derive the inner disc radius as $R_{\text {in }}=16.5_{-2.9}^{+1.5} D_{6}\left(\cos i / \cos 75^{\circ}\right)^{-1 / 2} \mathrm{~km}$ and $R_{\text {in }}=$ $111_{-6}^{+8} D_{6}\left(\cos i / \cos 75^{\circ}\right)^{-1 / 2} \mathrm{~km}$ (where $D_{6}$ is the distance in unit of $6 \mathrm{kpc}$ ) for the high/soft and 
low/hard state data, respectively. Thus, the inner radius in the low/hard state is $5.8-8.8$ times larger than that in the high/soft state, giving strong evidence for disc truncation in the low/hard state.

\section{Suzaku Observation of $4 \mathrm{U} 1630-472$ in the Very High State}

$4 \mathrm{U}-1630$ is a Galactic black hole candidate recently attracting a great deal of interest. On 2012 September 28, Doppler-shifted emission lines from baryonic jets were discovered in the XMM-Newton observation [[]]. We performed a Suzaku ToO observation of 4U 1630-472 in the very high state, 4 days after the discovery of the emission lines. We refer the readers to [1] for details.

Figure 5 presents the time-averaged Suzaku spectrum of 4U 1630-472. The emission lines detected 4 days before are disappeared in our data, suggesting the baryonic jets are transient events. We model the Suzaku spectrum with a multi-color disc, its Comptonisation and reflection. Here we use the dkbbfth model, which accounts for the energy coupling of disc and Comptonised corona. We include both thermal and non-thermal Comptonisation. The best-fit model and the residuals of the spectral fit are plotted in Figure [5.

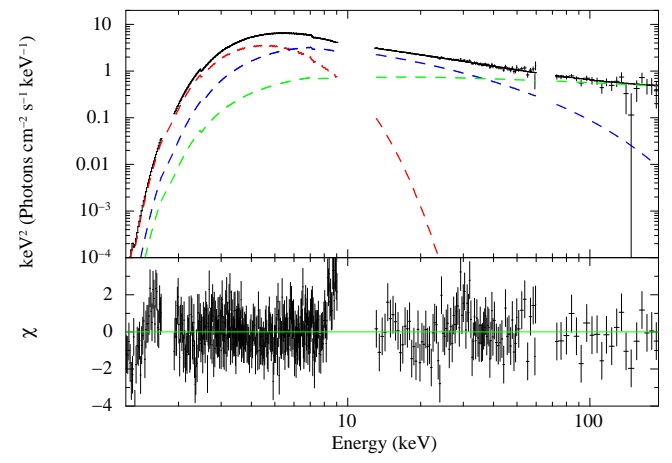

Figure 5: (Top) The time-averaged Suzaku spectrum of 4U 1630-472 and the best-fit model. The red, blue, green dashed lines indicate the contributions of the disc emission, thermal and non-thermal Comptonisation, respectively. Relativistic disc reflection is included in the Comptonisation components. (Bottom) Residuals of the spectral fit.

The inner disc radius obtained from this analysis is $R_{\text {in }}=41_{-1.7}^{+0.7} D_{10}\left(\cos i / \cos 70^{\circ}\right)^{-1 / 2} \mathrm{~km}$ (where $D_{10}$ is the distance in units of $\left.10 \mathrm{kps}\right)$, which is larger than that in the high/soft state $\left(R_{\text {in }}=\right.$ $\left.35 \pm 0.3 D_{10}\left(\cos i / \cos 70^{\circ}\right)^{-1 / 2} \mathrm{~km}\right)$, estimated from the data of the first observation of [प]]. This indicates that the standard disc in this state, as well as the low/hard state, recedes from the ISCO. Several recent studies report the same results ([0]; [B]; [ [18]) and therefore the disc truncation would be general in the very high state.

\section{Summary}

Our spectral analysis of the Suzaku and Swift data of H 1743-322, MAXI J1305-704, and $4 \mathrm{U} 1630-472$ suggests that the standard disc is truncated in the low/hard state and in the very high state. The low-frequency QPO detected in H 1743-322 is found to correlate the X-ray luminosity and photon index. This is consistent that the QPO is produced by Lense-Thirring precession of hot inner flow. 


\section{References}

[1] Chen, Y. P. et al., The 2009 outburst of H 1743-322 as observed by RXTE, 2010, A\&A, 522, 99

[2] Díaz Trigo, M. et al., Baryons in the relativistic jets of the stellar-mass black-hole candidate $4 U$ 1630-47, 2013, Nature, 504, 260

[3] Done, C., \& Kubota, A.,Disc-corona energetics in the very high state of Galactic black holes, 2006, MNRAS, 371, 1216

[4] Done, C. et al., Modelling the behaviour of accretion flows in X-ray binaries. Everything you always wanted to know about accretion but were afraid to ask, 2007, A\&ARv, 15, 1

[5] Hori T. et al., SUZAKU Observation of the Black Hole Binary 4U 1630-47 in the Very High State, 2014, ApJ, 790, 20

[6] Ingram, A. et al., Low-frequency quasi-periodic oscillations spectra and Lense-Thirring precession, 2009, MNRAS, 397, L101

[7] Kubota, A. et al., Evidence for a Black Hole in the X-Ray Transient GRS 1009-45, 1998, PASJ, 50, 667

[8] Kubota, A., \& Makishima, K., The Three Spectral Regimes Found in the Stellar Black Hole XTE J1550-564 in Its High/Soft State, 2004, ApJ, 601, 428

[9] Kubota, A., \& Done, C., The very high state accretion disc structure from the Galactic black hole transient XTE J1550-564, 2004, MNRAS, 353, 980

[10] Kubota, A. et al., Suzaku Discovery of Iron Absorption Lines in Outburst Spectra of the X-Ray Transient 4U 1630-472, 2007, PASJ, 59, 185

[11] Laor, A., Line profiles from a disk around a rotating black hole, 1991, ApJ, 376, 90

[12] Magdziarz, P., \& Zdziarski, A. A., Angle-dependent Compton reflection of X-rays and gamma-rays, 1995, MNRAS, 273, 837

[13] McClintock, J. E., \& Remillard, R. A., Black hole binaries, 2006, in Compact Stellar X-Ray Sources, ed. W. H. G., Lewin, \& M. van der Klis (Cambridge: Cambridge Univ. Press), 157

[14] Miller, J. M. et al., Swift Detection of Ionized X-ray Absorption in MAXI J1305-704, 2012, ATel \#4070

[15] Miller, J. M. et al., Chandra Spectroscopy of MAXI J1305-704: Detection of an Infalling Black Hole Disk Wind?, 2014, ApJ, 788, 53

[16] Mitsuda, K. et al., Energy spectra of low-mass binary X-ray sources observed from TENMA, 1984, PASJ, 36, 741

[17] Morihana, K. et al., MAXI/GSC Discovery of the Black-Hole Candidate MAXI J1305-704, 2013, PASJ, 65, L10

[18] Tamura., M. et al., The Truncated Disk from Suzaku Data of GX 339-4 in the Extreme Very High State, 2012, ApJ, 753, 65

[19] Shakura, N. I., \& Sunyaev, R. A., Black holes in binary systems. Observational appearance., 1973, A\&A, 24, 337

[20] Shidatsu, M. et al., The Accretion Disk and Ionized Absorber of the $9.7 \mathrm{hr}$ Dipping Black Hole Binary MAXI J1305-704, 2013, ApJ, 779, 26

[21] Shidatsu, M. et al., Spectral and Timing Properties of the Black Hole X-Ray Binary H 1743-322 in the Low/Hard State Studied with Suzaku, 2014, ApJ, 789, 100 